\title{
Ausgewählte Probleme der stilistischen Übersetzbarkeit von wissenschaftlichen Texten
}

\author{
Selected problems concerning translatability of \\ stylistic features in scientific writing
}

ABSTRACT. The dissemination of results has always been an important aspect of the research process. The translation plays here an increasingly important role. In view of the fact that the languages of science differ significantly, that the scientific style is simultaneously distinguished by many personal, universal and cultural features, the translation appears not to be an easy undertaking. Following the Galtung's concept of intellectual styles we present the distinguishing features of the Teutonic and Saxonic style on the example of German and English language.

KEYWORDS: scientific translation, scientific style, intellectual traditions, languages of science and their distinguishing features, translator training, Teutonic/Saxonic intellectual style, dialogic/ monologic communication, exposition/contemplation, linearity/digressiveness, beginning a new paragraph.

Die rasant fortschreitende wissenschaftliche Forschung und die technologische Entwicklung mancher Länder geschieht heutzutage vornehmlich im Rahmen einer internationalen Zusammenarbeit. Die enge Zusammenarbeit zwischen Wissenschaftlern verschiedener Nationen aber bringt die Notwendigkeit mit sich, die Forschungsergebnisse möglichst schnell zu veröffentlichen und einem anderssprachigen Publikum zugänglich $\mathrm{zu}$ machen. Im Prozess des Technologietransfers und des internationalen wissenschaftlichen Austausches spielt somit die Sprachmittlung eine zentrale Rolle. Die Übersetzungen sollen prinzipiell schnellstmöglich angefertigt werden, damit 
der Zugang zu den neuen Erkenntnissen in keiner Weise erschwert wird. Dementsprechend erweist es sich als notwendige Aufgabe, die zukünftigen Übersetzer auf die vielschichtigen Probleme der Übertragung wissenschaftlicher Inhalte aufmerksam zu machen.

Wie die Beschleunigung der Wissenschaftsentwicklung zustande kommt, lässt sich in erster Linie an einer steigenden Zahl von Wissenschaftlern und wissenschaftlichen Publikationen beobachten. Marszałek (1986: 43,65) vertritt in diesem Zusammenhang die Ansicht, dass die Zahl der Wissenschaftler und die Anzahl wissenschaftlicher Publikationen in diversen Ländern eine gleichbleibende Tendenz zur Verdoppelung binnen 7-15 Jahren aufweist. Barczaitis und Arntz (1998: 793f.) sehen die Ursache dieses Prozesses in dem im Bereich der Wissenschaft allgemein vorherrschenden Veröffentlichungs-, Rezeptions- und Kritikgebot.

Arbeitsergebnisse sollen schnell veröffentlicht werden, und die auf einem bestimmten Gebiet tätigen Fachleute haben wiederum ein Interesse daran, solche Veröffentlichungen mit geringstmöglicher Verzögerung zur Kenntnis zu erhalten, um darauf reagieren zu können. Das Übersetzen spielt hier eine große Rolle, denn trotz der (zunehmenden) Bedeutung des Englischen als Verständigungsmittel in der "Scientific community" werden doch immer wieder wichtige Veröffentlichungen in anderen Sprachen vorgenommen (Barczaitis, Arntz 1998: 793f.)

Die beiden oben angesprochenen Aspekte - die enorme Bedeutung der englischen Sprache in der Forschung, die damit verbundene vorherrschende Überzeugung, „die Spitzenforschung spreche englisch” (Markl 1986: 20), sowie die unaufhörlich in anderen Sprachen erscheinenden Publikationen zeugen von der Notwendigkeit der Übersetzung. Der heutzutage einfachste Weg zur Anerkennung auf internationaler Ebene führt durch das Gewirr der englischen Sprache. Diese Publikationssprache bildet auch eine Lösung für die Sprecher kleinerer Sprachen (Marszałek 1986: 54). Ammon ergründet die Ursache dieses Phänomens:

So sehr ist moderne Wissenschaft auf Kooperation durch Kommunikation angewiesen, dass nur Sprachen, die allen Wissenschaftlern vertraut sind, keine Barrieren für die wissenschaftliche Kommunikation bilden. Wegen der Interdisziplinarität moderner Wissenschaft sollten diese Sprachen idealiter sogar nicht nur allen Wissenschaftlern einer bestimmten Fachrichtung, sondern allen Wissenschaftlern überhaupt vertraut sein. (Ammon 1991: 212)

Verstanden als ein rezeptiver Prozess ist die Vertrautheit mit der englischen Sprache heutzutage in Forscherkreisen zu erwarten (Ammon 1998: VI). Die Forderung, dass alle Wissenschaftler ein einwandfreies, publikationsfähiges Englisch beherrschen, ähnelt aber schon der Forderung nach der 
Quadratur des Kreises. Ammon (1991: 274) bemerkt an einer anderen Stelle seiner Arbeit, dass die deutschsprachigen Wissenschaftler dazu nur in Ausnahmefällen fähig sind. Meistens sind sie doch auf sprachliche Hilfe angewiesen. Sie stehen damit aber nicht allein, weil die Situation in vielen anderen nicht englischsprachigen Ländern ähnlich aussieht. In Anlehnung an unsere obigen Ausführungen lässt sich feststellen, dass im Prozess der Verbreitung wissenschaftlicher Erkenntnisse die Übersetzung in die englische Sprache eine wichtige Rolle spielen muss. Der genannte Prozess ist aber nicht einseitig. Es werden weiterhin Übersetzungen in andere Publikationssprachen vorgenommen. Außerdem müssen wichtige wissenschaftliche Erkenntnisse auch denjenigen zugänglich gemacht werden, die das Englische nicht beherrschen. Die Übersetzung spielt somit in den Wissenschaften „naturgemäß" eine besondere Rolle (Stolze 1999: 15).

Die Übersetzung trägt de facto zur Beschleunigung der Wissenschaftsentwicklung bei, wobei die Beschleunigung der Wissenschaftsentwicklung auch zu einer zunehmenden Zahl von Übersetzungen führt (Marszałek 1986: 53). Die somit gesicherte wissenschaftliche Zusammenarbeit ist nach Kalverkämper (1992: 31) die Basis dafür, dass Europa ständig zusammenwächst und, dass „die internationalen Aktivitäten der Fachleute (...) eine wichtige friedenserhaltende Dimension (haben - K. J.)". Eine unpräzise oder sogar falsche Übersetzung aber kann letztlich vielleicht sogar das Gegenteil bewirken. Kozłowska (2007: 13 - üb: K.J.) betont, dass „der wissenschaftliche Text (...) ein besonderes Produkt der intellektuellen Tätigkeit eines Menschen (ist - K.J.). Der Inhalt eines solchen Textes muss ganzheitlich vermittelt werden" Die Arbeit des Übersetzers muss sich hier möglichst präzise und vorsichtig gestalten, damit es nicht zu Verständnisproblemen kommt, die heutzutage doch immer häufiger und sogar zwischen Experten ein und desselben Fachgebiets auftreten (Arntz, Picht 1995: 1). Die „Verschiedenheiten können nicht lediglich Kommunikationsstörungen veranlassen, sondern auch Vorurteile über Gruppen und Individuen, die menschliche Beziehungen (...) und den freien wissenschaftlichen Austausch beeinträchtigen" (Clyne 1993: 4).

Die Übersetzung wissenschaftlicher Texte stellt den Sprachmittler gemeinhin vor Herausforderungen auf verschiedenen Ebenen. Das Ausmaß dieser Schwierigkeiten führte schon seit Jahrhunderten manche Translationstheoretiker zur These von der Unübersetzbarkeit wissenschaftlicher Texte. Eine solche Auffassung vertrat auch der bedeutende Übersetzungstheoretiker Schleiermacher (1813). Laut ihm sind die philosophischen und poetischen Texte (in unserem heutigen Verständnis: „Texte der Wissenschaft und der Kunst" - Koller 2004: 43) diejenigen, die sich nicht übersetzen las- 
sen. Koller fasst die wichtigsten Punkte der Argumentation Schleiermachers folgendermaßen zusammen:

Texte der Wissenschaft und der Kunst (d.h. philosophische und poetische Texte) sind als unübersetzbar zu betrachten: hier ist das, was gesagt wird, und wie es sprachlich gefasst wird, auf einzelsprachspezifische Weise verbunden. Die Sprache ist nicht nur Vehikel von Inhalten, sondern sie ist selbst Inhalt bzw. determiniert diese Inhalte. Mit anderen Worten: Wenn man den betreffenden Text adäquat verstehen will, muss man in den "Geist der Sprache" eindringen, in das also, was in der Sprache selbst gedacht ist. (Koller 2004: 43)

Schleiermacher betont mit Recht die Einzelsprachspezifik wissenschaftlicher Texte, verabsolutiert aber Hindernisse, die mit ihrer Übersetzung verbunden sind. Dass die Wissenschaftstexte durch die in der gegebenen Sprache kodifizierte spezifische Weise der Weltbetrachtung determiniert sind, dem lässt sich nicht widersprechen. Alle Textarten stehen mehr oder weniger unter dem Einfluss, der in der sprachwissenschaftlichen Forschung im Rahmen der sog. "Sapir-Whorf-Hypothese” und des „Prinzips der sprachlichen Relativität" (Whorf 1956/2002) erörtert wird. Tabakowska (2001: 178 üb.: K.J.) betont in diesem Zusammenhang, dass "die Menschen, die unterschiedliche Sprachen sprechen, von den diversen grammatischen Kategorien dieser Sprachen dazu geneigt, und sogar gezwungen werden, die Welt auf eine spezifische Art und Weise zu betrachten". Dies spiegelt sich z.B. in den Benennungen für Begriffe wider (Scherer 1992: 28; Stolze 1999: 30; Tabakowska 2001: 185). Arntz und Picht (1995: 155) äußern die Auffassung, dass das Problem bei der mehrsprachigen Terminologiearbeit vor allem darin liegt, dass in den einzelnen Sprachen eine unterschiedliche begriffliche Gliederung der außersprachlichen Wirklichkeit erkennbar ist. Dementsprechend können Wörter "als sprachlich fixierte Theorien über die Welt aufgefasst werden" (Scherer 1992: 28).

Die von dem Wissenschaftler vorgenommene Wahl eines bestimmten Wortschatzes zeugt von seinem bevorzugten Stil. Dabei wird der Stil wissenschaftlicher Kommunikation durch mehrere Faktoren beeinflusst. Er trägt zugleich universelle, individuelle und kulturelle Merkmale. Somit ist die Übersetzung eines wissenschaftlichen Stils an sich kein leichtes Unterfangen.

Man darf nicht außer Acht lassen, dass die Übersetzung immer eine Art Kommunikation bildet (Vgl. Hejwowski 2006), wo jedes Merkmal eines ausgangssprachlichen Textes eine direkte oder versteckte Botschaft mitkommunizieren will. Das primäre Ziel der Translation besteht laut Żmudzki darin: 
Jeder Translationsakt soll - gemäß dem ursprünglichen Vorhaben des TranslationsInitiators - einigermaßen eine Kommunikation schaffen. Sie ist der Sinn jeder Translation und muss unter dem Blickwinkel dieser Kategorien betrachtet werden. (Żmudzki 2013: 179 - üb.: K.J.)

Den Kern eines solchen Kommunikationssystems bildet „ein Mensch Translator und zugleich Subjekt bestimmter kognitiv-kommunikativer Aktivitäten, der innerhalb eines Relationsnetzes mit den anderen natürlichen Objekten dieses Systems funktioniert" (Żmudzki 2013: 177 - üb.: K.J.). Als die übrigen Objekte zählt Kielar (2013: 5) den Ausgangstext, den Zieltext, den Primärsender, den Finalempfänger und den Initiator auf. Gemeinsam mit dem Übersetzer bilden sie die wichtigsten Stützpunkte für das Translationsmodell.

Mit Recht schlägt jedoch Żmudzki (2013: 181) eine erweiterte Version des Translationsmodells vor, in der folgende Objekte berücksichtigt weden: der Translations-Initiator, der Verfasser des Ausgangstextes, der Ausgangstext, der potentielle Benutzer des Ausgangstextes, der Translator, der Zieltext, der Zieltextrezipient, sowie der potentielle Benutzer des Zieltextes als Kommunikationsmultiplikator. Źmudzki erwähnt in seiner Auflistung praktisch alle beim Übersetzungsprozess beteiligten Kommunikationspartner, wobei er auch nicht vergisst, dass die immateriellen Faktoren einen ebenso großen Einfluss auf die Charakteristika der Übersetzung ausüben. Dem Translationsmodell muss, so Żmudzki (2013: 181), auch die Kategorie der Übersetzungsaufgabe hinzugefügt werden. An einer anderen Stelle seiner Arbeit erörtert er, aus welchen Komponenten eine solche Aufgabe besteht:

Um die Übersetzungsaufgabe konstruieren zu können, muss der Übersetzer mehrfach seine Auftragsangaben erweitern oder auch ergänzen. Dies betrifft in erster Linie die Bestimmung des Translationsziels, der vollen Charakteristik des Zieltextrezipienten, der institutionellen oder anderen Bedingungen, die die Verwirklichung der vermittelten Kommunikation determinieren. Dies bedeutet für den Übersetzer eine Notwendigkeit, Informationen durch verschiedene Kommunikationshandlungen zu gewinnen. (Żmudzki 2013: 183 - üb.: K.J.)

Auf diese unverzichtbare Informationen bezieht sich auch Kautz (2000: 49ff.), indem er in seinem Translationsmodell die übrigen immateriellen „Elemente (...) der textgebundenen Kommunikationshandlung Übersetzen” präsentiert. Dies sind laut ihm: Zeit, Ort, Kommunikationsanlass, Rezeptionsbedingungen, Intentionen des Ausgangstextverfassers und des Auftraggebers, Übersetzungsauftrag und Erwartungen des Zieltextrezipienten.

Das Problematische bei der Übersetzung wissenschaftlicher Texte liegt in erster Linie darin, dass hier die Intentionen des Ausgangstextverfassers und ggf. des Auftraggebers nicht unbedingt mit den Erwartungen des Zieltextre- 
zipienten in Einklang stehen müssen, hauptsächlich in Bezug auf stilistische Gestaltung des Textes. In Anbetracht dessen lässt sich feststellen, dass die wissenschaftlichen Texte von dem Übersetzer zunächst übersetzbar gemacht werden müssen. Die Aufgabe des Translators besteht in dieser Hinsicht darin, einen "mehrfachen Spagat” (Kautz 2000: 53) vollzuführen, um eine Balance zwischen den unterschiedlichen Intentionen und Erwartungen herzustellen.

Als das grundlegende Problem erweist sich in diesem Zusammenhang die Einschätzung, welche Züge eines gegebenen Textes von der kulturellen Färbung und welche von dem individuellen Kommunikationsstil zeugen. Tabakowska versucht das Wesen des Kommunikationsstils auf folgende Art und Weise zu ergründen:

Der individuelle Stil für das sprachliche Kommunizieren ist nicht rigoristisch vordeterminiert durch die kulturellen Skripte, die durch das Leben inmitten einer gegebenen Kultur erworben werden. Es gibt hier immer Platz für individuelle und gesellschaftliche Unterschiede, sowie für die Einführung von Änderungen. Der kommunikative Stil - sowohl einer Gesellschaft als auch eines Individuums - muss aber sowieso unter dem starken Einfluss „kultureller Regeln“ der Kommunikation bleiben. (Tabakowska 2001: 198, üb: K.J.)

Die Antwort auf die Frage, woran dieser Einfluss zu erkennen ist, erweist sich für die Übersetzer wissenschaftlicher Texte als sehr bedeutend. Die Anzeichen eines individuellen Kommunikationsstils sollen möglicherweise im zielsprachlichen Text aufrechterhalten werden, wobei man mit den Anzeichen einer kulturellen Färbung anders verfährt. Der Unterschied zwischen diesen Einflüssen ist nicht leicht zu erkennen, wenn man mit den Gegebenheiten der anderen Kultur nicht vertraut ist. Dementsprechend kommt dem Ausbildungsprozess eine wichtige Funktion zu: „seine Aufgabe ist es, die Wechselbeziehungen zwischen der ununterbrochenen aktiven Auseinandersetzung mit der fremden Kultur und der Qualität der Übersetzung aufzuzeigen" (Jopkiewicz 2013: 2, üb: K.J.). Der Prozess der Ausbildung zukünftiger Übersetzer wissenschaftlicher Texte muss auch notwendigerweise typische Merkmale der wissenschaftlichen Sprache und konkrete Symptome für die kulturelle Prägung erkennbar machen.

Macpherson (2012: 22) äußert sich folgendermaßen zu den unterschiedlichen Vorstellungen von dem Wesen der wissenschaftlichen Sprache: „as with any type of language there are as many different conceptions of the academic register as there are people who use it. This is not to say that certain features cannot be isolated that many might consider typical or even representative of it". Und gerade diese typischen Merkmale der wissenschaftlichen Ausdrucksweise wollen wir im Folgenden in Bezug auf zwei 
bedeutende Träger der wissenschaftlichen Kommunikation: Englisch und Deutsch, analysieren.

Wissenschaftliche Texte weisen in verschiedenen Sprachen im Allgemeinen viele Gemeinsamkeiten auf. Fleischmann und Schmitt (2011: 534) stellen fest, dass man sogar bis etwa 1980 von der Annahme ausging, das wissenschaftliche Denken sei universell, der wissenschaftliche Diskurs - kulturunabhängig. Es hat sich aber im Laufe der Zeit herausgestellt, dass sich die Wissenschaftssprachen in manchen Aspekten auch deutlich voneinander unterscheiden. Die oben angeführte Entdeckung verdanken wir Galtung (1981/1985/2000), der die These aufgestellt hat, dass es außer universellen und individuellen Zügen der wissenschaftlichen Kommunikation noch die kulturellen Merkmale gibt, welche von der Existenz eines im Rahmen einer bestimmten Kultur erzeugten intellektuellen Stils zeugen. Die Forscher (Clyne 1996; Duszak 1997a; 1997b; Kozłowska 2007; Kußmaul 2011; Fleischmann, Schmitt 2011; Lehman 2013) unterscheiden in Anlehnung an die Feststellungen von Galtung (1981/1985/2000) zwischen vier intellektuellen Stilen: dem teutonischen, sachsonischen, gallischen und nipponischen Stil. Die zwei zunächst erwähnten Stile erweisen sich als wichtig für unsere Analyse, da sie typisch für die deutsche und englische Sprache sind.

Kozłowska (2007: 35) weist auf manche Ähnlichkeiten und Gemeinsamkeiten zwischen der russischen, tschechischen und deutschen Sprache hin, deren intellektueller Stil als teutonisch bezeichnet wird. Zum teutonischen Stil wird von manchen Forschern (Duszak 1997b; Lehman 2013) auch die polnische Sprache gezählt. Der sachsonische Stil wird demgegenüber von Kozłowska (2007: 35) als typisch für die englische Sprache charakterisiert. In der Forschung wird in diesem Zusammenhang vor allem das Deutsche (als das repräsentativste Beispiel des teutonischen Stils) dem Englischen gegenübergestellt. Duszak (1997b) unternimmt ebendiesen Versuch, indem sie die groben Merkmale dieser zwei intellektuellen Stile erörtert:

In reporting research, writers may adopt a style that is more or less reader-oriented (Hinds 1987). Sensitivity to readers' needs is sometimes discussed under dialogic versus monologic, or expository versus contemplative tendencies in academic narration (...). The dialogic formula, apparently typical of Anglo-American academic texts, is believed to be more interactiv and hence more reader-friendly. Such an effect stems from, among other things, reader guidance and discourse predictability through staging and signposting. As a result, a piece of academic writing turns into a reciprocally negotiable contract, in which the reader participates in setting the pace and the line of exposition rather than dutifully following the writer's road to delivery. This, in turn, contrasts with a kind of contemplative rhetoric that is traditionally combined with Teutonic intellectual traditions (...). Here writers are believed to in- 
dulge more in acts of creative thinking, and to endeavor more to reproduce them in the name of science and for the sake of truth, than to report them. (Duszak 1997b: 13)

Kozłowska (2007: 35) scheint die obigen Ausführungen unbeabsichtigt zu bestätigen, indem sie die weiteren ergänzenden Merkmale des teutonischen und sachsonischen Stils zur Sprache bringt. Der teutonische Stil ist ihr zufolge kompliziert, die argumentative Ausführung - verwickelt. In diesem Zusammenhang betont die Forscherin das häufige Vorkommen von Nominalisierungen, Schachtelsätzen und Satzellipsen. Als charakteristisch für den teutonischen Stil erweise sich, so Kozłowska, auch seine Digressivität, die sich in vielen Rückverweisen, Ergänzungen, näheren Bestimmungen und Wiederholungen manifestiere. Die Digressivität ist eine Art Antwort auf die von Clyne (1993: 11) gestellte Frage nach der Verktorialität des wissenschaftlichen Textes: „In welche geometrische Richtung bewegt sich der Text fort?” Die Ausführungen in den Texten deutschsprachiger Wissenschaftler weisen nämlich die Tendenz einer mehrsträngigen Argumentation auf, während die englischsprachigen Texte nach Linearität (Clyne 1993: 11) streben. Clyne bezieht sich in seiner Arbeit auf die Konnotationen, die in oben genannten Sprachräumen an die entsprechenden Formen der Vektorialität gebunden werden:

Während sowohl englisch- wie die deutschsprachige Wissenschaftler Texte herstellen, die einen größeren oder kleineren Grad der Linearität zeigen, enthalten die deutschen Texte eine größere Zahl von Exkursen/'Abweichungen'. Werden digressions im Englischen vielfach negativ eingeschätzt, so haben Exkurse im deutschen Texten spezifische Funktionen. In den von uns analysierten Texten ermöglichen sie dem Autor, zusätzlichen Inhalt einzuschieben, eine theoretische Perspektive einzufügen, eine historische Übersicht zu verschaffen oder dem Text eine ideologische Dimension zu geben. Ferner erlauben die Exkurse eine Polemik mit Vertretern anderer Schulen. (Clyne 1993: 12)

Aus obigen Ausführungen lässt sich schlussfolgern, dass die für die deutschsprachigen Texte charakteristische Digressivität im englischsprachigen Raum a priori negativ konnotiert wird. Angemessen scheint es uns an dieser Stelle, der primären Ursache dieser wissenschaftlichen Sichtweise auf den Grund $\mathrm{zu}$ gehen. Einen ersten Hinweis finden wir im Beitrag von Ylönen (1993: 84). Die Wissenschaftlerin äußert die Auffassung, dass lineare Texte schneller rezipiert und die enthaltenen Informationen - schneller gefunden werden können. Es ist nicht besonders kompliziert, die direkte Hinwendung in Richtung des Rezipienten an dieser Äußerung zu erkennen. Dies bildet auch ein weiteres Merkmal des sachsonischen Stils. Die englischsprachigen Texte sind in erster Linie empfängerorientiert, die deutschsprachigen Texte konzentrieren sich vor allem auf den Autor selbst und auf 
das zu vermittelnde Wissen. Auf die Frage: „wer trägt die Verantwortung für die erfolgreiche Verständigung in wissenschaftlicher Kommunikation?" hält Clyne folgende Antwort bereit:

Im Deutschen und Japanischen, zwei Sprachkulturen mit inhaltlicher Orientierung, trägt der Leser die Verantwortung, den Text zu verstehen, der von demjenigen stammt, der das Wissen zu vermitteln hat, d.h. die ungleichen Sozialbeziehungen sollen hervorgehoben werden. Der Nachdruck fällt auf Verständnis. Im Englischen hingegen, wo es sich mehr um Verstehen handelt, muss der Autor sich bemühen, einen lesbaren Text herzustellen, wenn er die darin enthaltenen Argumente und Informationen verbreiten will. (Clyne 1993: 9)

Die Rezeptionsprozesse können weitgehend erleichtert werden, indem die in den Ausführungen präsentierten Gedanken nicht dichtgedrängt, sondern in gewisser Weise getrennt voneinander stehen. Plaxco gibt englischsprachigen Wissenschaftlern hoc sensu einige Hinweise zur Erstellung guter Texte an die Hand:

A paragraph should discuss only a single idea and thus should have a single, unifying theme running throughout it. (...) Flow is an equally critical element of good paragraph structure: every sentence in a paragraph should arise logically from the sentence before it, and transition logically into the sentence that follows. (Plaxco 2010: 2263).

Im Verständnis von Plaxco ist ein guter wissenschaftlicher Text ein einfacher und - im Sinne der Linearität - klar strukturierter Text. Bei einer solchen Strukturierung erweist sich die Verwendung sog. thematischer Sätze (engl. 'topic sentences') am Anfang eines jeden Absatzes als besonders hilfreich. Thematische Sätze sind besonders typisch für Ausführungen linearer Art (Ylönen 1993: 84) und somit auch für den sachsonischen Stil. Ihre Aufgabe besteht darin, in den Hauptinhalt eines gegebenen Absatzes einzuführen (Fiedler 2012: 45) und das neue Thema anzukündigen (Ylönen 1993: 87). Hingegen bevorzugt es der teutonische Stil, mehrere Gedankenstränge in mehreren Textabsätzen zugleich anzusprechen und miteinander zu verflechten. $\mathrm{Zu}$ diesem Zwecke werden in deutschsprachigen Texten die sog. Brükkensätze (engl. bridge sentences) verwendet. Sie stehen zumeist am Anfang eines neuen Absatzes und erfüllen eine Bezugsfunktion. Sie nehmen Bezug auf vorangehende oder frühere Textabschnitte (Clyne 1991: 55; Ylönen 1993: 87) oder - wenn sie sich am Ende eines Absatzes befinden - auf den nachfolgenden Absatz (Fiedler 2012: 45).

Clyne unternimmt den Versuch, den prozentualen Wert der thematischen und aufzählenden Sätze, sowie der Brückensätze in der wissenschaftlichen Kommunikation innerhalb des englischsprachigen und deutschsprachigen Kulturraumes zu ermitteln: 
Tab. 1. Verwendung bestimmter Typen von Sätzen in wissenschaftlichen Texten (Clyne 1991: 55)

$\begin{array}{lll} & \text { ENGLISH-SPEAKING } & \text { GERMAN-SPEAKING } \\ \text { Topic sentences } & 62 \% & 48 \% \\ \text { Enumerating sentences } & 38 \% & 23 \% \\ \text { Bridge sentences } & - & 29 \% \\ & \text { English texts } & \text { German texts } \\ & & \\ \text { Topic sentences } & 30 \% & 57 \% \\ \text { Enumerating sentences } & 40 \% & 14 \% \\ \text { Bridge sentences } & 30 \% & 29 \%\end{array}$

Die oben genannten prozentualen Werte belegen die Tatsache, dass die englischsprachigen Wissenschaftler eine starke Tendenz zur Verwendung thematischer und aufzählender (wie z.B. 'There are a number of...' - Clyne 1991: 55) Sätze aufweisen, wohingegen die deutschsprachigen Forscher dies viel seltener tun. Hingegen machen die Texte englischsprachiger Wissenschaftler keinen Gebrauch von der Möglichkeit der Verwendung von Brükkensätzen, welche sich wiederum im deutschsprachigen Raum großer Beliebtheit erfreuen.

Vielmehr erweist es sich, dass die englischsprachigen Texte, die von deutschen Wissenschaftlern verfasst werden, genauso viele Brückensätze enthalten, wie ein von denselben Wissenschaftlern verfasster muttersprachlicher Text. Bestimmt bleibt diese Situation nicht ohne negative Auswirkungen auf die globale Rezeption der Texte im englischsprachigen Kulturraum. Schon darin ist ein deutlicher Beweis dafür zu sehen, dass die „interkulturellen Unterschiede in der Vertextung" (Trumpp 1998: 40) den zukünftigen Übersetzern notwendigerweise beigebracht werden sollen, denn „wer die sprachspezifischen Konventionen erhalten und befremdliche Wirkungen vermeiden will, muss darauf achten, dass er nicht muttersprachliche Konventionen auf den in der Fremdsprache abzufassenden Text überträgt" (Stolze 1999: 114).

Unsere obigen Ausführungen resümierend wollen wir ein Verzeichnis von Merkmalen zweier intellektueller Stile vorschlagen. Die unten vorgeschlagene Liste erhebt keinen Anspruch auf Vollständigkeit. Ihr primäres Ziel besteht lediglich darin, die Grundprinzipien wissenschaftlicher Kommunikation in beiden Sprachen zu ermitteln und somit den zukünftigen Übersetzern wissenschaftlicher Texte als erste Orientierung zu dienen. 
Tab. 2. Merkmale des sachsonischen und teutonischen Stils

\begin{tabular}{|c|c|c|}
\hline Merkmale & Sachsonischer Stil & Teutonischer Stil \\
\hline Typ der Interaktion & Dialogizität & Monologizität \\
\hline Einstellung des Autors & Exposition & Kontemplation \\
\hline $\begin{array}{l}\text { Argumentative Ausfüh- } \\
\text { rung }\end{array}$ & einfach & mehrsträngig \\
\hline $\begin{array}{l}\text { Gedankengang des } \mathrm{Au}- \\
\text { tors }\end{array}$ & leicht $\mathrm{zu}$ folgen & schwierig zu folgen \\
\hline $\begin{array}{l}\text { Verantwortung für erfolg- } \\
\text { reiche Verständigung }\end{array}$ & Autor des Textes & Rezipient des Textes \\
\hline $\begin{array}{l}\text { Umgang mit den Informa- } \\
\text { tionen }\end{array}$ & Bericht & Reproduktion \\
\hline Vektorialität & Linearität & Diggressivität \\
\hline $\begin{array}{l}\text { Arten der Absatzeröff- } \\
\text { nung }\end{array}$ & $\begin{array}{l}\text { thematische und aufzäh- } \\
\text { lende Sätze }\end{array}$ & Brückensätze \\
\hline $\begin{array}{l}\text { Anzahl der Gedanken pro } \\
\text { Absatz }\end{array}$ & Einzelgedanke & Gedankengeflecht \\
\hline
\end{tabular}

Die wissenschaftliche Kommunikation folgt den strengen Regeln unterschiedlicher Sprachen, Kulturen und letztendlich auch Stile. Der Ausbildungsprozess hat somit die Aufgabe, zukünftigen Sprachmittlern Hilfsmittel an die Hand zu geben, auf den ersten Blick nicht sichtbare Prinzipien aufzudecken, um diese erfolgreich bei der Übersetzung wissenschaftlicher Texte einzusetzen.

\section{LITERATURVERZEICHNIS}

Ammon, U., 1991. Die internationale Stellung der deutschen Sprache. Berlin-New York: de Gruyter.

Ammon, U., 1998. Ist Deutsch noch internationale Wissenschaftssprache? Berlin-New York: de Gruyter.

Arntz, R., Picht, H., 1995. Einführung in die Terminologiearbeit. Hildesheim-Zürich-New York: Georg Olms.

Barczaitis, R., Arntz, R., 1998. Fachübersetzung in den Naturwissenschaften und in der Technik. In: Hoffman, L., Kalverkämper, H., Wiegand, H.E. (Hrsg.). Fachsprachen. Ein internationales Handbuch zur Fachsprachenforschung und Terminologiewissenschaft. Berlin-New York: de Gruyter, 792-800. 
Clyne, M., 1991. The Sociocultural Dimension: The Dilemma of the German-speaking Scholar. In: Schröder, H. (Hrsg.). Subject-oriented Texts: Languages for Special Purposes and Text Theory. Berlin-New York: de Gruyter, 49-67.

Clyne, M., 1993. Pragmatik, Textstruktur und kulturelle Werte. Eine interkulturelle Perspektive. In: Schröder, H. (Hrsg.). Fachtextpragmatik. Tübingen: Narr, 3-18.

Clyne, M., 1996. Inter-cultural Communication at Work: Cultural Values in Discourse. Cambridge-New York: Cambridge University Press.

Duszak, A., 1997a. Analyzing digressiveness in Polish academic texts. In: Duszak, A. (Hrsg.). Culture and styles of academic discourse. Berlin-New York: de Gruyter, 323-342.

Duszak, A., 1997b. Cross-cultural academic communication: a discourse-community view. In: Duszak, A. (Hrsg.). Culture and styles of academic discourse. Berlin-New York: de Gruyter, 11-40.

Fiedler, S., 2012. Zur Kulturspezifik der Wissenschaftskommunikation. In: Brosch, C., Fiedler, S. (Hrsg.). Fackkommunikation - interlinguistische Aspekte. Beiträge der 21. Jahrestagung der Gesellschaft für Interlinguistik e.V., 18. - 20. November 2011 in Berlin. Berlin: GIL, 39-50.

Fleischmann, E., Schmitt, P.A., 2011. Fachsprachen und Übersetzung. In: Kittel, H., House, J., Schultze, B. (Hrsg.). Übersetzung: Translation: Traduction. HSK 26.1. Berlin: de Gruyter, 531-542.

Galtung, J., 1985/2000. Struktur, Kultur und intellektueller Stil. Ein vergleichender Essay über sachsonische, teutonische, galische und nipponische Wissenschaft. In: Wierlacher, A. (Hrsg.). Das Fremde und das Eigene: Prolegomena zu einer interkulturellen Germanistik. München: iudicium, 151-193. (1981. Structure, culture and intellectual style. An essay comparing saxonic, teutonic, gallic and nipponic approaches).

Hejwowski, K., 2006. Kognitywno-komunikacyjna teoria przekładu. Warszawa: PWN.

Jopkiewicz, K., 2013. Język to nie wszystko: wiedza kulturo- $i$ realioznawcza a jakość przekładu ustnego. <http://www.konferencje-spnjo.polsl.pl/download/jopkiewicz-k-jezyk-tonie-wszystko-wiedza-kulturo-i-realioznawcza-a-jakosc-przek.pdf> (abgerufen am: 15.02.2014).

Kalverkämper, H., 1992. Die kulturanthropologische Dimension von 'Fachlichkeit' im Handeln und Sprechen. Kontrastive Studien zum Deutschen, Englischen, Französischen, Italienischen und Spanischen. In: Albrecht, J., Baum, R. (Hrsg.). Fachsprache und Terminologie in Geschichte und Gegenwart. Tübingen: Narr, 31-58.

Kautz, U., 2000. Handbuch Didaktik des Übersetzens und Dolmetschens. München: Iudiucium.

Kielar, B.Z., 2013. Zarys translatoryki. Warszawa: IKL@.

Koller, W., 2004. Einführung in die Übersetzungswissenschaft. Wiebelsheim: Quelle \& Meyer.

Kozłowska, Z., 2007. O przekładzie tekstu naukowego (na materiale tekstów językoznawczych). Warszawa: WUW.

Kußmaul, P., 2011. Die Übersetzung gesisteswissenschaftlicher Texte aus sprachwissenschaftlicher Perspektive. In: Kittel, H., House, J., Schultze, B. (Hrsg.). Übersetzung: Translation: Traduction. HSK 26.1. Berlin: de Gruyter, 636-641.

Lehman, I.M., 2013. Rhetorical approaches to academic writing: the case of polish and angloamerican academic writing. <http://www.retoryka.edu.pl/files/far2_2013_art5.pdf> (abgerufen am: 18.02.2014). 
Macpherson, R., 2012. English for Academic Purposes. Warszawa: PWN.

Markl, H., 1986. Die Spitzenforschung spricht englisch. In: Kalverkämper, H., Weinrich, H. (Hrsg). Deutsch als Wissenschaftssprache. Tübingen: Narr, 20-25.

Marszałek, L., 1986. Edytorstwo publikacji naukowych. Warszawa: PWN.

Plaxco, K.W., 2010. The art of writing science. <http://www.smb.wsu.edu/docs/defaultsource/wotd/the-art-of-writing-science.pdf?sfvrsn=2> (abgerufen am: 19.02.2014).

Scherer, H., 1992. Kosmos - Sprache - Fachsprache. In: Albrecht, J., Baum, R. (Hrsg.). Fachsprache und Terminologie in Geschichte und Gegenwart. Tübingen: Narr, 17-30.

Schleiermacher, F., 1813. Ueber die verschiedenen Methoden des Uebersetzens. <http://users. unimi.it/dililefi/costazza/programmi/2006-07/Schleiermacher.pdf> (abgerufen am: 19.02.2014).

Stolze, R., 1999. Die Fachübersetzung: eine Einführung. Tübingen: Narr.

Tabakowska, E., 2001. Kognitywne podstawy języka i jezzykoznawstwa. Kraków: TAiWPN Universitas.

Trumpp, E.C., 1998. Fachtextsorten kontrastiv: Englisch - Deutsch - Französisch. Tübingen: Narr.

Whorf, B.L., 2002. Jezzyk, myśl i rzeczywistość. Warszawa: Wydawnictwo KR (1956. Language, Thought and Reality).

Ylönen, S., 1993. Stilwandel in wissenschaftlichen Artikeln der Medizin. Zur Entwicklung der Textsorte "Originalarbeiten" in der Deutschen Medizinischen Wochenschrift von 1884 bis 1989. In: Schröder, H. (Hrsg.). Fachtextpragmatik. Tübingen: Narr, 81-98.

Żmudzki, J., 2013. Holizm funkcjonalny w perspektywie translatoryki antropocentry cznej. In: Lingwistyka Stosowana 8, 177-187. 
